\title{
A Brief Review of Some Approaches to Hysteresis in Viscoelastic Polymers
}

\author{
H.T. Banks \\ Center for Research in Scientific Computation \\ North Carolina State University \\ Raleigh, NC 27695-8205
}

January 27, 2008

On the Occasion and in Honor of Professor V. Lakshmikantham's 84th Birthday Anniversary

\begin{abstract}
We give a brief review of hysteresis in viscoelastic polymers. The efforts surveyed range from phenomenological to molecular modelling with applications involving recent efforts on elastomers to biotissue.
\end{abstract}

\section{Introduction}

In control and systems theory, delay or hereditary systems or systems with memory (hysteresis) have played an important role for many years because of the early realizations by Minorsky and others $[40,41,56,57,58,60]$ that feedback design based on dynamics wherein one ignores any delays may fail catastrophically to stabilize or control a system in which delays or hysteresis are present in the dynamics. This is true whether the hysteresis is a fundamental part of the underlying dynamics or a part of the input or control operator. For the latter there is a growing body of literature [11, 12, 13, 45, 54, 68] on the Preisach and related theories for hysteretic control input such as arises in smart material systems $[27,39,64]$. Applications in which delays and hysteresis play a basic role in the underlying dynamics include sustained efforts in biology with early efforts employing delay systems $[1,2,16,17,28,29,30,31,32,42,51,53,55,62]$ and more recent investigations involving hysteretic probabilistic structures $[5,7]$ as well as classical materials and electromagnetics research (see [8] and the references therein). These applications drove a substantial amount of mathematical and computational research on hysteretic systems in the last half of the 20th century, e.g., see [26, 40, 41, 46, 47] among the many books, research monographs, and research articles written. Here we shall focus on the delays or hysteresis arising in the fundamental dynamics of the systems to be stabilized or controlled. In particular we consider viscoelastic materials that are polymeric in nature. This includes a wide range of materials 
of current importance such as (rubber or silicone based) filled elastomers and all types of biotissue (soft tissue, ligaments, cartilage, etc.).

\section{Viscoelasticity}

The mathematical modelling of viscoelasticity (sometimes also loosely referred to as hysteresis) in materials using ideas from elasticity has attracted the attention of a large number of investigators over the past century. Among significant contributors (see the many references in $[33,34,35,37,38,52,59,61,65,67,69,70])$ have been some of the true giants from the fields of engineering and material sciences. One of the most widely used empirical models for viscoelasticity in materials is the Boltzmann convolution law [22, 35, 37, 38, 69], one form of which is given in equation (1)

$$
\sigma(t)=g_{e}(\epsilon(t))+C_{D} \dot{\epsilon}(t)+\int_{-\infty}^{t} Y(t-s) \frac{d}{d s} g_{v}(\epsilon(s), \dot{\epsilon}(s)) d s
$$

where $\epsilon$ is the infinitesimal strain, $Y$ is the convolution memory kernel, and $g_{e}$ and $g_{v}$ are nonlinear functions accounting for the elastic and viscoelastic responses of the elastomers, respectively; for summaries and further references, see Chapter 2 of [38] as well as [22]. This form of model, when incorporated into force balance laws, results in integro-partial differential equations which are most often phenomenological in nature as well as being computationally challenging both in simulation and control design. This stress-strain law implies that the stress depends not only on the current strain and strain rate but also on the history of the strain and the strain-rate. It is very important to note that the stress-strain law (1) contains various standard internal strain or internal variable formulations as special cases. The anelastic displacement field (ADF) models of Lesieutre [48, 49] for composite materials exhibiting both elastic and anelastic displacement fields are formulated on the assumption that the host elastic material contains anelastic materials with internal strains $\varepsilon_{1}$ which are elastic strain driven. That is, the constitutive laws have the form

$$
\sigma(t)=E \varepsilon(t)-E_{1} \varepsilon_{1}(t)
$$

where the internal strain is given by

$$
\dot{\varepsilon}_{1}(t)+\frac{1}{\tau} \varepsilon_{1}(t)=c_{2} \varepsilon(t), \quad \varepsilon_{1}(0)=0,
$$

or equivalently,

$$
\varepsilon_{1}(t)=\int_{0}^{t} c_{2} e^{-\frac{t-s}{\tau}} \varepsilon(s) d s .
$$

Several generalizations of this formulation exist, e.g., Johnson, et al., [43, 44], suggest that the internal strain is strain rate driven, i.e.,

$$
\dot{\varepsilon}_{1}(t)+\frac{1}{\tau} \varepsilon_{1}(t)=c_{2} \dot{\varepsilon}(t)
$$


The Boltzmann-type law (1) (under appropriate assumptions on the past memory from $-\infty$ to 0$)$ corresponds to an internal strain model of the form

$$
\dot{\varepsilon}_{1}(t)+\frac{1}{\tau} \varepsilon_{1}(t)=\frac{d}{d t} g_{v}(\varepsilon(t), \dot{\varepsilon}(t)), \quad \varepsilon_{1}(0)=0 .
$$

This form is often chosen since one finds that neither (3) nor (4) provide laws that readily describe experimental data, especially in the cases of filled elastomers, biotissues and other molecular polymers.

\section{Fung's Quasi-linear Models}

Fung, in his extensive efforts [38] with biomechanics and biotissue, develops and presents the quasi-linear viscoelastic constitutive equation

$$
S_{i j}(t)=\int_{-\infty}^{t} G_{i j k l}(t-\tau) \frac{\partial S_{k l}^{(e)}[\bar{E}(\tau)]}{\partial \tau} d \tau,
$$

where $S_{i j}$ is the Kirchoff stress tensor, $\bar{E}$ is the Green's strain tensor, $G_{i j k l}$ is a reduced relaxation function, and $S_{k l}^{(e)}$ is the "elastic" stress tensor. For the scalar components $G_{i j k l}$, Fung proposes the reduced relaxation function $G(t)$ given in the form

$$
G(t)=\left\{1+C\left[E_{1}\left(\frac{t}{\tau_{2}}\right)-E_{1}\left(\frac{t}{\tau_{1}}\right)\right]\right\}\left[1+c \ln \left(\frac{\tau_{2}}{\tau_{1}}\right)\right]^{-1} .
$$

Here $E_{1}(z)=\int_{z}^{\infty} \frac{e^{-t}}{t} d t, C$ represents the degree to which viscous effects are present, and $\tau_{1}$ and $\tau_{2}$ represent fast and slow viscous time phenomena. We note that the internal strain variable formulation (2), (5) is equivalent to the constitutive relationship proposed by Fung if one considers an approximation of the relaxation function $G$ by a sum of exponential terms. Various internal strain variable models are investigated in [3] and a good agreement is demonstrated between a two internal strain variable model (e.g., of the form $\sigma=E \varepsilon-$ $\left.E_{1} \varepsilon_{1}-E_{2} \varepsilon_{2}\right)$ and undamped simulated data based on the Fung kernel $G$.

Since its introduction, this quasi-linear viscoelastic (QLV) theory of Fung has been applied successfully in stress-strain experiments to several types of biological tissue. A benefit to using (6) as a constitutive equation is that, unlike simpler models for viscoelasticity, it allows for the consideration of a continuous spectrum (e.g., see the discussions in [38]) of relaxation times and frequencies (this is also true of the probabilistic-based internal variable approach developed in [23] and described below). (The need for a continuum of relaxation times in certain materials was observed many years ago [36, 63, 66, 70].) While Fung's theory has been successfully employed for fitting hysteretic stress-strain curves, for control applications one is interested in using it in a full dynamical model. Unfortunately, the QLV, as presented by Fung, leads to exceedingly difficult computations within full dynamical partial differential equations, especially in estimation and control problems. This motivated the development of the internal variable approach described in $[3,23,48]$ (which permits discrete approximation to a continuum) in attempts to approximate well the corresponding dynamic responses even in cases where the stress-strain curves alone do not produce adequate approximations - see [38]. 
The probabilistic based internal variable alternative [23] to Fung's kernel involves a parameter dependent kernel with a continuous distribution of parameters and internal variables. In the case of a finite combination of Dirac $\delta$ distributions, one obtains a finite summation of exponential functions as the approximation kernel (see the discussions below). This method can be extended to allow for consideration of a continuous spectrum of relaxation times and frequencies by utilizing absolutely continuous parameter distributions in place of the $\delta$ distributions.

\section{Internal Variables}

The internal variable approach to overcome both conceptual and computational challenges is consistent with the belief that hysteresis is actually a manifestation of the presence of multiple scales in a physical or biological material system that is frequently modelled (and masked) with a phenomenological representation such as an hysteresis integral for the macroscopic stress-strain constitutive law. The internal variable modelling leads to an efficient computational alternative for the corresponding integro-partial differential equation models. In addition, it provides a "molecular" basis for the models (for a comparison of models of viscoelastic damping via hysteretic integrals versus internal variable representations, see [22] and the references therein).

Our own interest in viscoelasticity in polymeric materials has been motivated by projects in our Industrial Applied Mathematics Program with at least two of our industrial partners: The Lord Corporation and Medacoustics, Inc. The collaborations with polymer scientists and engineers at Lord involved the dynamic modelling of filled rubbers which experimentally exhibit both significant hysteresis and nonlinearity in tensile and shear deformations as depicted in the sample stress-strain curves in Figure 1. The efforts with engineers at Medacoustics used some of the viscoelastic models we have investigated in attempts to understand the propagation of arterial stenosis induced shear waves in composite biotissue in a sensor development and characterization project.

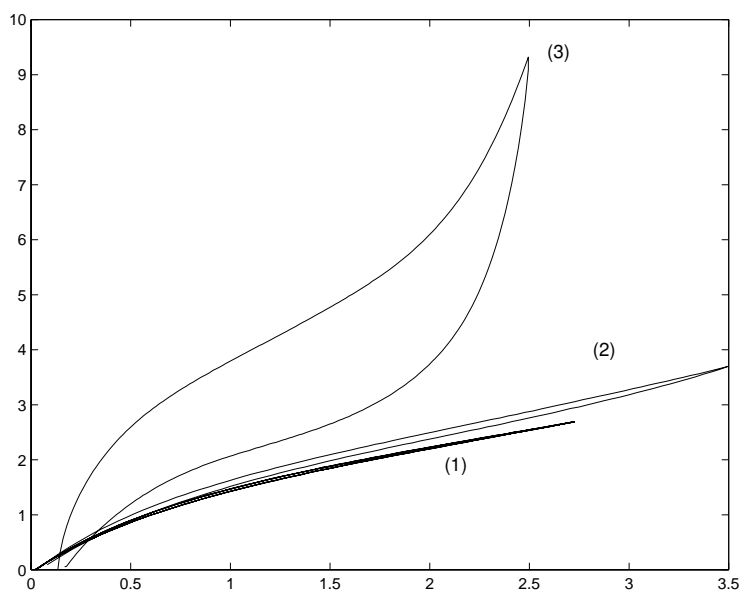

Figure 1: Experimental stress-strain curves for (1) unfilled, (2) lightly filled and (3) highly filled rubber in tensile deformations. 
In some of our earlier efforts [24, 25], the models for hysteretic damping in elastomers employed a phenomenological Boltzmann-type constitutive law of the form (1). As explained in $[20,24]$, our nonlinear materials undergoing large deformations required the use of finite (as opposed to infinitesimal) strain theories [61]. However, since the nonlinearity between the stress and finite strain is an unknown to be estimated (using inverse problem algorithms) and since the finite strain can be expressed in terms of known nonlinearities as a function of the infinitesimal strain (at least in the problems of interest here), one can effectively formulate the problem as one of estimating the unknown nonlinearity between stress and infinitesimal strain (see [24]). Hence one can develop models for stress in terms of infinitesimal strain. Our previous efforts as summarized in $[20,21]$ have shown, through comparison with experimental data, that the best fit to filled elastomer data occurs when $g_{e}$ and $g_{v}$ are cubic, along with $Y$ as a distribution of decaying exponentials. We subsequently [18, 19, 20, 21] developed nonlinear models based on stick-slip "molecular" ideas of Johnson and Stacer [43] and Doi and Edwards [34] which resulted in a form for $g_{e}, g_{v}$ and $Y$ in (1) that matched the empirical findings reported in [20, 24, 25]. These models allow for multiple relaxation times present in polymer strands of composite materials within a virtual compartmental model of entangled chemically cross-linked/physically constrained system of long chain "molecules". While accounting for multiple relaxation parameters, these models do not include physically or chemically based parameters in representations of the polymer strands.

We mention briefly two recent advances: $(i)$ a new constitutive model [10] that has been developed which combines the virtual stick-slip continuum "molecular-based" ideas of Johnson and Stacer [43] with the Rouse bead chain (see Figure 2 below) ideas as described in Doi and Edwards [34]; ( $i$ i) a two dimensional version [14, 15, 50] of a model that accounts for stenosis driven shear wave propagation in biotissue.

\section{$5 \quad$ A Stick-Slip/Rouse Hybrid Model}

The early models and the nonlinear extensions of the Boltzmann law did not provide insight into the underlying mechanisms for tensile and/or shear deformations in filled rubber or biotissue. This is not unexpected since the approaches described above are based on pseudophenomenological formulations. We then [19, 20, 21] turned to a different approach based on molecular arguments which, as we shall see, lead precisely to the class of models based on a Boltzmann hysteresis formulation. As usual, one begins with force and moment balance and seeks constitutive laws for the viscoelastic stress term $\sigma_{v i s c o}$ in

$$
\sigma(t ; \tau)=\sigma_{\text {elast }}(\varepsilon, \dot{\varepsilon})+\sigma_{\text {visco }}\left(\varepsilon_{1}(\cdot)\right)
$$

where $\varepsilon=\frac{\partial u}{\partial x}$ is the infinitesimal strain and $\varepsilon_{1}$ is an "internal strain" variable on which $\sigma_{v i s c o}$ depends in an hysteretic manner. As described above, we found that a reasonable description of the data of interest could be given with the typical stress-strain relationship

$$
\sigma(t)=g_{e}(\varepsilon(t), \dot{\varepsilon}(t))+\int_{0}^{t} \gamma e^{-\frac{t-s}{\tau}} \frac{d}{d s} g_{v}(\varepsilon(s), \dot{\varepsilon}(s)) d s
$$


where $\tau$ is a relaxation parameter, $g_{v}$ is defined with cubic polynomials and $g_{e}=g_{e}\left(\frac{\partial u}{\partial x}\right)+$ $C_{D} \frac{\partial^{2} u}{\partial t \partial x}$. We have already observed that this expression is equivalent to

$$
\sigma(t)=\tilde{g}_{e}(\varepsilon(t), \dot{\varepsilon}(t))+\gamma \varepsilon_{1}(t ; \tau),
$$

where, for a given "relaxation parameter" $\tau$, the internal strain $\varepsilon_{1}(t ; \tau)$ satisfies (5). In fact, we found that highly filled rubbers required multiple relaxation times $\tau_{1}, \tau_{2}$ in an approximation to (7) to obtain good model fits to the data. As one might expect, molecular based formulations, where microscopic relaxation parameters vary across the population of molecules in the material, lead to internal dynamics of the form (5),(8) that involve multiple values of $\tau$. When combined with a Prohorov metric framework (see [4,6]) for uncertainty in internal dynamics, these ideas lead to the computational models we have used. Indeed, the molecular based approach leads to a general class of models with uncertainty or randomness in the stress

$$
\sigma(t, x ; P)=\tilde{g}_{e}(\varepsilon(t, x), \dot{\varepsilon}(t, x))+\gamma \int_{\mathcal{T}} \varepsilon_{1}(t, x ; \tau) d P(\tau),
$$

where $P$ is a probability distribution over the set $\mathcal{T}$ of possible relaxation parameters, and $\varepsilon_{1}(t ; \tau)$ satisfies, for each $\tau \in \mathcal{T}$,

$$
\dot{\varepsilon}_{1}(t, x ; \tau)+\frac{1}{\tau} \varepsilon_{1}(t, x ; \tau)=\dot{\varepsilon}(t, x) h(\varepsilon(t, x)) .
$$

For the reptation model derivation in [19], one begins with the Doi/Edwards [34] stick-slip molecular models as embodied in the continuous tube reptation models of Johnson/Stacer [43] wherein polymer materials such as rubber are postulated to be composed of two types of molecules. In tensile deformations, one denotes by $L(t)$ the length of chemically cross-linked or CC molecules, while $\ell(t)$ denotes the length of physically constrained or PC molecules. To use stick-slip models in continuum simulations of reptation in rubbers, one considers networks of "cells" or boxes of parallel-sided CC boxes and PC boxes with sides of length (principal stretches)

$$
\lambda_{c}=1+\varepsilon=1+\frac{\partial u_{c}}{\partial x}, \quad \lambda_{p}=1+\varepsilon_{1}=1+\frac{\partial u_{p}}{\partial x},
$$

respectively. Here $u_{c}$ denotes the deformations of the $\mathrm{CC}$ box and $u_{p}$ denotes the deformations of the PC box. Using a linear stick-slip assumption as in [43], and strain energy densities based on experiments of Young and Danik (see [18, 20] for details), one obtains as a limit of $\mathrm{PC}$ response to step tensile deformations of the $\mathrm{CC}$ molecules, the $\varepsilon, \varepsilon_{1}$ coupled dynamics

$$
\dot{\varepsilon}_{1}+\frac{1}{\tau} \varepsilon_{1}=\dot{\varepsilon} \frac{1+\varepsilon_{1}}{1+\varepsilon} .
$$

However, if one replaces the linear assumption of [18] by a nonlinear stick-slip hypothesis (which is the basis of the work in [19]), one obtains a more general nonlinear, dynamical relationship between $\varepsilon$ and $\varepsilon_{1}$ given by

$$
\dot{\varepsilon}_{1}+\frac{1}{\tau} \varepsilon_{1}=\dot{\varepsilon} f\left(\left(1+\varepsilon_{1}\right) /(1+\varepsilon)\right) .
$$


Expansion and truncation of higher order terms lead to equations of the form

$$
\dot{\varepsilon}_{1}+\frac{1}{\tau} \varepsilon_{1}=\dot{\varepsilon}\left(\alpha_{0}+\alpha_{1} \varepsilon+\alpha_{2} \varepsilon^{2}+\alpha_{3} \varepsilon^{3}\right),
$$

which are of the same form as the internal variable model $(5),(8)$ with $g_{v}$ a cubic polynomial. For the corresponding contributions to $\sigma$ from the strain energy densities of Young-Danik/ Johnson-Stacer with the nonlinear stick-slip hypothesis, one obtains a contribution to the rate independent strain $g_{v}^{s}$ (after expanding $f$ in a Taylor series and dropping higher order terms) of the form

$$
g_{v}^{s}\left(\varepsilon, \varepsilon_{1}\right)=g_{\text {cubic }}(\varepsilon)+\gamma_{1} \varepsilon_{1},
$$

where $\varepsilon_{1}$ is as before (i.e., the internal strain satisfying (10)). Thus, the total stress-strain relationship can be written in the form (9). If the measure $\mathrm{P}$ of (9) has atoms at $\tau_{1}$ and $\tau_{2}$, (i.e., the measure is composed of Dirac measures concentrated at $\tau_{1}$ and $\tau_{2}$ ), then the constitutive law leads precisely to the model

$$
\sigma(t, x ; P)=\tilde{g}_{e}(\varepsilon(t, x), \dot{\varepsilon}(t, x))+\gamma_{1} \varepsilon_{1}\left(t, x ; \tau_{1}\right)+\gamma_{2} \varepsilon_{2}\left(t, x ; \tau_{2}\right),
$$

which was used in the data fits in $[3,20]$. Further details on this modeling approach can be found in $[9,21]$

We next developed a new constitutive model which combines the "molecular-based" ideas of Johnson and Stacer with the Rouse bead chain ideas and explained its relation to the Boltzmann phenomenological models.

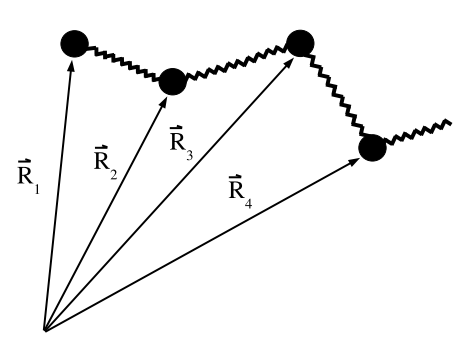

Figure 2: Representation of vectors for a bead-spring polymer molecule.

The new molecular-based constitutive model, in which polymer chains are treated as Rouse type strings of interconnected beads (a reasonable approximation for many materials), permits the incorporation of many important physical parameters (such as temperature, segment bond length, internal friction, and segment density) in the overall hysteretic constitutive relationship. Its form is similar to that developed in $[19,20]$ and does have the general form (1) of Boltzmann type, even though the kernel is not of convolution type. The resulting model does, however, provide a molecular basis for the earlier pseudo-phenomenological stick-slip models.

We give only a brief outline of the new constitutive model here; more details of the derivation can be found in the report [10]. We model a polymer material undergoing directional deformation by assuming it is composed of two virtual compartments as depicted in Figure 3. 


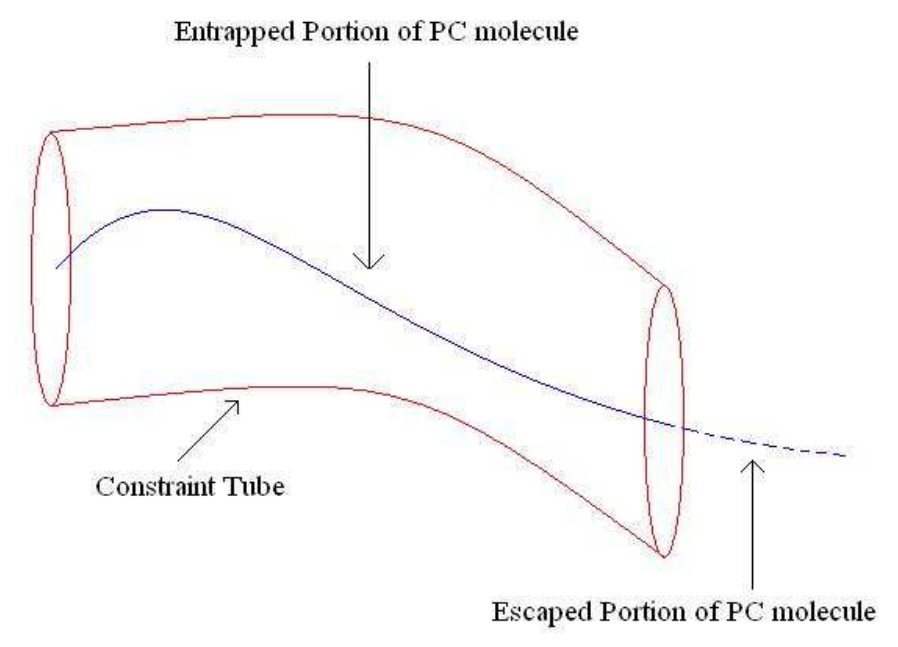

Figure 3: PC molecule entrapped by the surrounding constraining tube.

One compartment consists of a constraining tube which is a macroscopic compartment containing both CC (chemically cross-linked) and PC (physically constrained) molecules. The other compartment is microscopic in nature and consist of those PC molecules aligned with the direction of the deformation. These molecules will at first "stick" to the constraining tube and be carried along with its motion, but will very quickly "slip" and begin to "relax" back to a configuration of lower strain energy. In the model derivation one computes the contributions of both "compartments" to the overall stress of this polymer material undergoing deformations to obtain the constitutive law.

\section{Stenosis-Driven Shear Wave Propagation in Biotis- sue}

Finally, we turn to recent results on the viscoelastic models for propagation of stenosis-driven biotissue waves mentioned above. In the biotissue efforts $[14,15,50]$, we employ an internal variable formulation of Boltzmann type hysteresis laws to investigate the propagation of stenosis generated waves in biotissue where it has been demonstrated that a viscoelastic (as opposed to an elastic) formulation is important and that waves generated in a twodimensional cylindrical geometry with inner radius partial occlusions can be readily modelled and simulated.

Specifically we have carried out efforts on two and three dimensional models that employ an internal variable approach to model wave propagation. To motivate this, we recall [3] that coronary artery disease (CAD) is caused by atherosclerosis, the gradual accumulation of plaque along the walls of an artery. This buildup, known as a stenosis, restricts the flow of blood, leading to a decrease in the oxygen supply to the heart muscle. It is well known that 
arterial stenoses produce sounds due to turbulent blood flow in partially occluded arteries. In principle, turbulent normal wall forces exist at and downstream from an arterial stenosis, exerting pressure on the wall of the artery which then causes a small displacement in the surrounding body tissue. The goal is to model the propagation of the wave generated from the stenosis to the chest wall, and ultimately, to create an inverse problem methodology which can be utilized to determine the location of an arterial stenosis. In $[14,15,50]$ we also discuss comparison of the viscoelastic model to an elastic one as well as present typical simulations for a biologically motivated example.

\section{Acknowledgements}

This research was supported in part by the US Air Force Office of Scientific Research under grant AFOSR-FA9550-04-1-0220.

\section{References}

[1] H.T. Banks, Modeling and Control in the Biomedical Sciences, Lecture Notes in Biomath., Vol. 6, Springer-Verlag, Berlin, Heidelberg, New York, 1975.

[2] H.T. Banks, Delay systems in biological models: approximation techniques, Nonlinear Systems and Applications (V. Lakshmikantham, ed.), Academic Press, New York, 1977, 21-38.

[3] H.T. Banks, J.H. Barnes, A. Eberhardt, H. Tran and S. Wynne, Modeling and computation of propagating waves from coronary stenoses, Computational and Applied Mathematics, 21 (2002), 767-788.

[4] H.T. Banks and K. Bihari, Modeling and estimating uncertainty in parameter estimation, CRSC-TR99-40, NCSU, December 1999; Inverse Problems, 17 (2001), 1-17.

[5] H.T. Banks, D.M. Bortz and S.E. Holte, Incorporation of variability into the modeling of viral delays in HIV infection dynamics, CRSC-TR01-25, November, 2001; Math Biosci., 183 (2003), 63-91.

[6] H.T. Banks, D. Bortz, G.A. Pinter and L.K. Potter, Modeling and imaging techniques with potential application in bioterrorism, CRSC-TR03-02, NCSU, January 2003; Chapter 6 in Bioterrorism: Mathematical Modeling Applications in Homeland Security (H.T. Banks and C. Castillo-Chavez, eds.), Frontiers in Applied Mathematics, Vol.28, SIAM, Philadelphia, 2003, 129-154.

[7] H.T. Banks, S. Dediu and H.K.Nguyen, Time delay systems with distribution dependent dynamics, CRSC-TR06-15, May, 2006; IFAC Annual Reviews in Control, 31 (2007), $17-$ 26. 
[8] H.T. Banks and N.L. Gibson, Electromagnetic inverse problems involving distributions of dielectric mechanisms and parameters, CRSC-TR05-29, NCSU, August, 2005; Quarterly of Applied Mathematics, 64 (2006), 749-795.

[9] H.T. Banks, J.B. Hood and N.G. Medhin, A molecular based model for polymer viscoelasticity: Intra- and inter-molecular variability, CRSC-TR04-39, December, 2004; Applied Mathematical Modelling, to appear.

[10] H.T. Banks, J.B. Hood, N.G. Medhin and J.R. Samuels, A stick-slip/Rouse hybrid model for viscoelasticity in polymers, Technical Report CRSC-TR06-26, NCSU, November, 2006; Nonlinear Analysis: Real World Applications, to appear (available online doi:10.1016/j.nonrwa.2007.06.015).

[11] H.T. Banks and A.J. Kurdila, Hysteretic control influence operators representing smart material actuators: Identification and approximation, CRSC-TR96-23, August, 1996; Proc. 35th IEEE Conf. on Decision and Control (Kobe, Japan), December, 1996, 37113716.

[12] H.T. Banks, A.J. Kurdila and G. Webb, Identification of hysteretic control influence operators representing smart actuators, Part I: Formulation , CRSC-TR96-14, April 1996; Mathematical Problems in Engineering, 3 (1997), 287-328.

[13] H.T. Banks, A.J. Kurdila and G. Webb, Identification of hysteretic control influence operators representing smart actuators: Part II, Convergent approximations , CRSCTR97-7, April, 1997; J. of Intelligent Material Systems and Structures, 8 (1997), 536550.

[14] H.T. Banks and N.S. Luke, Simulations of propagating shear waves in biotissue employing an internal variable approach to dissipation, Technical Report CRSC-TR06-28, NCSU, December, 2006; Communications in Computational Physics, 3 (2008), 603-640.

[15] H.T. Banks, N. S. Luke and J. R. Samuels, Jr., Viscoelasticity in polymers: Phenomenological to molecular mathematical modelling, CRSC-TR06-29, December, 2006; Numerical Methods for Partial Differential Equations, 23 (2007), 817-831.

[16] H.T. Banks and J.M. Mahaffy, Global asymptotic stability of certain models for protein synthesis and repression, Quart. Applied Math., 36 (1978), 209-221.

[17] H.T. Banks and J.M. Mahaffy, Stability of cyclic gene models for systems involving repression, J. Theoretical Biology, 74 (1978), 323-334.

[18] H.T. Banks and N.G. Medhin, A molecular based dynamic model for viscoelastic responses of rubber in tensile deformations, CRSC-TR00-27, NCSU, October, 2000; Communications on Applied Nonlinear Analysis, 8 (2001), 1-18.

[19] H.T. Banks, N.G. Medhin and G.A. Pinter, Nonlinear reptation in molecular based hysteresis models for polymers, Quarterly Applied Math., 62 (2004), 767-779. 
[20] H.T. Banks, N.G. Medhin and G.A. Pinter, Multiscale considerations in modeling of nonlinear elastomers, Technical Report CRSC-TR03-42, NCSU, October, 2003; J. Comp. Meth. Engr. Sci. and Mech., 8 (2007), 1-10.

[21] H.T. Banks, N.G. Medhin and G.A. Pinter, Modeling of viscoelastic shear: A nonlinear stick-slip formulation, CRSC-TR06-07, February, 2006; Dynamic Systems and Applications, to appear.

[22] H.T. Banks and G.A. Pinter, Damping: hysteretic damping and models, CRSC-TR9936, NCSU, December, 1999; in Encyclopedia of Vibration ( S.G. Braun, D. Ewins and S. Rao, eds.), Academic Press, London, 2001, 658-664.

[23] H.T. Banks and G.A. Pinter, A probabilistic multiscale approach to hysteresis in shear wave propagation in biotissue, Multiscale Modeling and Simulation, 3 (2005), 395-412.

[24] H.T. Banks, G.A. Pinter, L.K. Potter, M.J. Gaitens and L.C. Yanyo, Modeling of quasistatic and dynamic load responses of filled viscoelastic materials, Technical Report CRSC-TR98-48, NCSU, December, 1998; Chapter 11 in Mathematical Modeling: Case Studies from Industry (E. Cumberbatch and A. Fitt, eds.), Cambridge University Press, 2001, 229-252.

[25] H.T. Banks, G.A. Pinter, L.K. Potter, B.C. Muñoz and L.C. Yanyo, Estimation and control related issues in smart material structures and fluids, Technical Report CRSCTR98-02, NCSU, January, 1998; Optimization Techniques and Applications (L. Caccetta, et al., eds.), Curtain Univ. Press, July, 1998, 19-34.

[26] R. Bellman and K.L. Cooke, Differential-Difference Equations, Academic Press, New York, 1963.

[27] M. Brokate and J. Sprekels, Hysteresis and Phase Transitions, Springer-Verlag, New York, 1996.

[28] S.N. Busenberg and K.L. Cooke, eds., Differential Equations and Applications in Ecology, Epidemics, and Population Problems, Academic Press, New York, 1981.

[29] V. Capasso, E. Grosso and S.L. Paveri-Fontana, eds., Mathematics in Biology and Medicine, Lecture Notes in Biomath., Vol. 57, Springer-Verlag, Berlin, Heidelberg, New York, 1985.

[30] J. Caperon, Time lag in population growth response of Isochrysis Galbana to a variable nitrate environment, Ecology, 50 (1969), 188-192.

[31] K.L. Cooke, Functional differential equations: Some models and perturbation problems, in Differential Equations and Dynamical Systems (J.K. Hale and J.P. LaSalle, eds.), Academic Press, New York, 1967, 167-183.

[32] J.M. Cushing, Integrodifferential Equations and Delay Models in Population Dynamics, Lec. Notes in Biomath., Vol. 20, Springer-Verlag, Berlin and New York, 1977. 
[33] M. Doi, Introduction to Polymer Physics, Clarendon Press, Oxford, 1996.

[34] M. Doi and M. Edwards, The Theory of Polymer Dynamics, Oxford, New York, 1986.

[35] J.D. Ferry, Viscoelastic Properties of Polymers, John Wiley and Sons, Inc., New York, 1961.

[36] J.D. Ferry, E.R. Fitzgerald, L.D. Grandine and M.L. Williams, Temperature dependence of dynamic properties of elastomers: relaxation distributions, Ind. Engr. Chem., 44 (1952), 703-706.

[37] Y.C. Fung, Foundations of Solid Mechanics, Prentice-Hall, Englewood Cliffs, NJ, 1965.

[38] Y.C. Fung, Biomechanics: Mechanical Properties of Living Tissues, Springer-Verlag, New York, 1993.

[39] M.V. Gandhi and B.S. Thompson, Smart Materials and Structures, Chapman and Hall, London, 1992.

[40] A. Halanay, Differential Equations, Academic Press, New York, 1966.

[41] J.K. Hale, Functional Differential Equations, Springer-Verlag, New York, 1971.

[42] F.C. Hoppensteadt, ed., Mathematical Aspects of Physiology, Lectures in Applied Math, Vol. 19, American Mathematical Society, Providence, 1981.

[43] A.R. Johnson and R.G. Stacer, Rubber viscoelasticity using the physically constrained system's stretches as internal variables, Rubber Chemistry and Technology, 66 (1993), $567-577$.

[44] A.R. Johnson, C.J. Quigley and J.L. Mead, Large strain viscoelastic constitutive models for rubber, part I: Formulations, Rubber Chemistry Technology, 67 (1994), 904-917.

[45] M.A. Krasnosel'skii and A.V. Pokrovskii, Systems with Hysteresis, Nauka, Moscow, 1983; translated, Springer-Verlag, Berlin, 1989.

[46] V. Lakshmikantham and M. Rama Mohana Rao, Theory of Integro-Differential Equations, Gordon \& Breach Science Publ., New York, 1995.

[47] V. Lakshmikantham, Lizhi Wen and B.G. Zhang, Theory of Differential Equations with Unbounded Delay, Kluwer Academic Publ., Dordrecht, The Netherlands, 1994.

[48] G.A. Lesieutre, Modeling frequency-dependent longitudinal dynamic behavior of linear viscoelastic long fiber components, J. Composite Materials, 28 (1994), 1770-1782.

[49] G.A. Lesieutre and K. Govindswamy, Finite element modeling of frequency-dependent and temperature-dependent dynamic behavior of viscoelastic materials in simple shear, Int. J. Solids Structures, 33 (1996), 419-432.

[50] N.S. Luke, Modeling Shear Wave Propagation in Biotissue: An Internal Variable Approach to Dissipation, Ph.D. dissertation, NC State University, August, 2006. 
[51] N. MacDonald, Time lag in a model of a biochemical reaction sequence with end-product inhibition, J. Theor. Biol., 67 (1977), 727-734.

[52] J.E. Marsden and T.J.R. Hughes, Mathematical Foundations of Elasticity, Prentice-Hall, Englewood Cliffs, NJ, 1983.

[53] M. Martelli, K.L. Cooke, E. Cumberbatch, B. Tang and H. Thieme, eds., Differential Equations and Applications to Biology and to Industry, World Scientific, Singapore. 1996.

[54] I.D. Mayergoyz, Mathematical Models of Hysteresis, Springer-Verlag, New York, 1991.

[55] J.A.J. Metz and O. Diekmann, eds., The Dynamics of Physiologically Structured Populations, Lecture Notes in Biomath., Vol. 68, Springer-Verlag, Berlin, Heidelberg, New York, 1986.

[56] N. Minorsky, Self-excited oscillations in dynamical systems possessing retatded actions, J. Applied Mechanics, 9 (1942), A65-A71.

[57] N. Minorsky, On non-linear phenomenon of self-rolling, Proc. National Academy of Sciences, 31 (1945), 346-349.

[58] N. Minorsky, Nonlinear Oscillations, Van Nostrand, New York, 1962.

[59] R.W. Ogden, Non-Linear Elastic Deformations, Ellis Horwood Limited, Chichester, 1984.

[60] M.N. Oguztorelli, Time-Lag Control Systems, Academic Press, New York, 1966.

[61] R.S. Rivlin, Large elastic deformations of isotropic materials, I, II, III, Phil. Trans. Roy. Soc. A, 240 (1948), 459-525.

[62] K. Schmitt, ed., Delay and Functional Differental Equations and Their Applications, Academic Press, New York, 1972.

[63] F. Schwarzl and A.J. Staverman, Higher approximation methods for the relaxation spectrum from static and dynamic measurements of viscoelastic materials, Appl. Sci. Res., A4 (1953), 127-141.

[64] R.C. Smith, Smart Material Systems: Model Development, Frontiers in Applied Mathematics, Vol. FR32, SIAM, Philadelphia, 2005.

[65] J.K. Stille, Introduction to Polymer Chemistry, John Wiley and Sons, Inc., New York, 1962.

[66] D. Ter Haar, A phenomenological theory of viscoelastic behavior, Physica, 16 (1950), 839-850.

[67] L.R.G. Treloar, The Physics of Rubber Elasticity, Clarendon, Oxford, 1975. 
[68] A. Visitin, Differential Models of Hysteresis, Springer-Verlag, New York, 1994.

[69] I.M. Ward, Mechanical Properties of Solid Polymers, J. Wiley \& Sons, New York, 1983.

[70] M.L. Williams and J.D. Ferry, Second approximation calculations of mechanical and electrical relaxation and retardation distributions, J. Poly. Sci., 11 (1953), 169-175. 